\title{
Interference in Argon Auger Spectra
}

\author{
D. K. Waterhouse and J.F. Williams \\ Centre for Atomic, Molecular and Surface Physics, Physics Department, The University of Western Australia, \\ Nedlands, Perth, WA 6907, Australia
}

(Received 25 November 1996)

\begin{abstract}
Angle-dependent interference was observed in $(e, 2 e)$ coincidence observations of argon $L_{2,3} M_{2,3} M_{2,3}$ Auger transitions. The $(e, 2 e)$ dynamics include an incident energy of $1.0 \mathrm{keV}$, a fast electron scattering angle of $21^{\circ}$ for an energy range from 730 to $760 \mathrm{eV}$ with $2.5 \mathrm{eV}$ resolution and Auger electron detection angles of $60^{\circ}$ and $90^{\circ}$. Observations were made below the Auger threshold and with excess energies of 22,2 , and $0.5 \mathrm{eV}$. The interference, attributed to the interaction of Auger and satellite-state ionization processes, and the post-collision-interaction effects are quantified. [S0031-9007(97)03545-X]

PACS numbers: $34.80 . \mathrm{Dp}$
\end{abstract}

The occurrence and significance of interference effects [1] in Auger decay, and particularly their $(e, 2 e)$ coincidence observations [2], have aroused considerable interest. We report new and intriguing aspects of the interference effects that occur below the Auger threshold and when the excess energy of the ejected electron is small. The argon atom is particularly suited to $(e, 2 e)$ coincidence observations of these effects because of the relative energies of the Auger $L_{2,3} M_{2,3} M_{2,3}{ }^{1} S_{0},{ }^{1} D_{2}$, and ${ }^{3} P_{0,1,2}$ transitions and the inner- and outer-shell ionization processes. An incident electron energy of $1.0 \mathrm{keV}$ and a scattered electron detection angle of $21^{\circ}$ determined the kinematics for which the $(e, 2 e)$ satellite coincidence intensity at $60^{\circ}$ is a maximum, and is about a factor of 20 higher than the intensity at $90^{\circ}$. These scattering conditions correspond, respectively, to a large continuum background, enabling the study of interference effects, or a small background for which interference effects were negligible and postcollision-interaction (PCI) effects could be studied. We report the first observations of the strong angular behavior of the interference phenomena, and the quantified behavior of the interference and PCI effects for energies below the inner-shell ionization threshold, and for excess energies up to $22 \mathrm{eV}$ above threshold.

The origin of PCI effects can be illustrated as follows. After the Auger electron is produced during inner-shell decay, the ejected electron sees a singly charged core until, for selected scattering dynamics, it is overtaken by the Auger electron. Then the ejected electron sees a doubly charged core and slows down in the stronger field while the Auger electron then sees a singly ionized core and gains energy. The effects of PCI have been modeled for electron impact [3] and observed in many noncoincidence experiments [4]. Limited $(e, 2 e)$ coincidence data for selected transitions have been reported for low excess energies [2,5-8]. Those experiments aimed to determine how PCI affected the angular dependence of Auger transition intensities, and also to measure the PCI effects which are observable as a shift in the energy distribution of the Auger electron and asymmetric broadening of the line shape. The magnitude of the energy shift and line shape broadening increases as the excess energy is decreased. The triple differential cross sections that include PCI effects for Auger transitions [3] are given by

$$
\frac{d^{6} \sigma}{d E_{s} d E_{A} d \Omega_{s} d \Omega_{A}}=\frac{\Gamma / 2 \pi}{\varepsilon_{a}^{2}+\Gamma^{2} / 4} \sigma\left(E_{s}, \Omega_{s}\right) K\left(\varepsilon_{a}, \bar{\xi}\right),
$$

where $\Gamma$ quantifies the Auger electron lifetime and $\varepsilon_{a}=$ $E-E_{A}$ is the difference between the observed electron energy $E$ and the nominal Auger transition energy $E_{A}$. The $L_{2,3}$ ionization cross section is given by $\sigma\left(E_{s}, \Omega_{s}\right)$. The function $K\left(\varepsilon_{a}, \bar{\xi}\right)$ accounts for the particle interactions. The parameter $\bar{\xi}$ quantifies the Coulomb interaction between different final state electrons and is velocity dependent. The combination of a rapid variation in $K(\varepsilon, \bar{\xi})$ across an Auger transition linewidth due to its dependence on the ratio $\varepsilon_{a} / \Gamma$, and its exponential dependence on the velocity term $\bar{\xi}$ [3], leads to strong distortion of the Lorentz profile for low excess energies.

The presence of interference effects in Auger coincidence data for the argon $L_{3} M_{2,3} M_{2,3}{ }^{1} D_{2}$ transitions was indicated [8] when data, at $2 \pm 0.9 \mathrm{eV}$ excess energy and an Auger electron detection angle of $70^{\circ}$, were fitted better by a Fano line shape given by [9]

$$
\sigma_{\mathrm{obs}}=\sigma_{1}(q+\varepsilon)^{2}\left(\varepsilon^{2}+1\right)^{-1}+\sigma_{2} .
$$

This form of the observed cross section $\sigma_{\text {obs }}$ indicates the contributions from the cross section $\sigma_{1}$, arising from the continuum that interferes with the resonant transition, and the cross section $\sigma_{2}$ of the noninteracting continuum. Also $\varepsilon$ differs from $\varepsilon_{a}$ (above) by a normalization factor of $1 / \Gamma$. Here the "resonant" states are the Auger products of electron-impact ionization of the argon $L_{2,3}$ inner shells. The ratio of the symmetric and asymmetric parts of the Auger transition amplitude is given by the " $q$ " parameter. Because of the small coincidence signals and the difficulty of these experiments, previous workers observed small interference effects in the argon $L_{3} M_{2,3} M_{2,3}{ }^{1} S_{0}[6]$ and ${ }^{1} D_{2}$ 
[8] line shape, but did not determine the angle or excess energy dependence. They proposed the interfering process was direct double ionization. In contrast, we have found the dominant interference mechanism appears to be the outer-shell satellite state single ionization process, particularly when the excess energy is low. As well, this Letter shows conclusively the angle dependence, and quantifies the interference effects for the argon $L_{2,3} M_{2,3} M_{2,3}{ }^{1} S_{0}$, ${ }^{1} D_{2}$, and ${ }^{3} P_{0,1,2}$ transitions for a range of excess energies.

The essential feature of the apparatus that enables this coincidence experiment is a multiparameter coplanar $(e, 2 e)$ coincidence spectrometer [10]. Microchannel-plate detectors on the output of each of two hemispherical electrostatic energy analyzers allow the simultaneous collection of data for a large range of excess energies with good energy resolution for more than one entire Auger transition line shape. In that way the excess energy dependence of PCI and interference effects can be observed for a number of Auger transitions simultaneously. The excess energy resolution was $\pm 1.3 \mathrm{eV}$ and the Auger detector resolution was $420 \mathrm{meV}$ at $60^{\circ}$ and $500 \mathrm{meV}$ at $90^{\circ}$. The $L_{2,3} M_{2,3} M_{2,3}$ Auger line shapes in the absence of PCI effects are Lorentzian with a linewidth of about $120 \mathrm{meV}$. The relative intensity of each Auger transition was determined from the noncoincidence data which were measured simultaneously with the coincidence signal. The residual shift of Auger transitions measured in the noncoincidence spectra is expected to be about $14 \mathrm{meV}$ [11], and this was taken into consideration when the Auger electron energy scales were determined. In the coincidence data the continuum background varies slowly in the region of the Auger transitions and is formed by the coincidence signals from ionization of outer-shell satellite states [12].

During the data analysis of the separate measurements at $60^{\circ}$ and $90^{\circ}$ it became apparent that neither a PCI model nor a Fano line shape model could fit both data sets with similar accuracy. Each parameter in the PCI model [3] was predetermined by the experimental conditions of each data set. The only adjustable parameters were the continuum background level and an overall scaling factor for the Auger transition amplitudes.

In the Fano model the only adjustable parameters were the continuum background level, the $q$ parameter, and a scaling factor for each of the $L_{2}$ and $L_{3}$ initial vacancy states. Initially PCI was neglected, but as the PCI effects are expected to be isotropic, it is important to include those effects in the Fano model. This was done by including a shift in the nominal Auger transition energy of $\Delta \varepsilon_{a}=$ $\bar{\xi} \Gamma / 2$, with $\bar{\xi}$ and $\Gamma$ as defined earlier, and by using a Fano linewidth that was consistent with the full width at half maximum of the predicted PCI-broadened line shape [3]. The combined model gives a more realistic indication of the amount of interference and usually resulted in a better fit, except below threshold, and this will be discussed shortly. Before comparison with the experimental data the theoretical line shapes were convoluted with a suitable Gaussian spectrometer function in each model.
The comparison of our data and fits for the same excess energies at different angles enable the influence of interference and PCI effects to be compared easily. Figures $1(a)-1$ (c) and 2(a) -2(c) are a series of such comparisons in which the excess energy for the $L_{3}$ transitions is noted in the top left of each plot. The excess energy for the $L_{2}$ transitions is about $2.2 \mathrm{eV}$ smaller. Figure 1 contains coincidence data recorded with the Auger detector at $90^{\circ}$, and in Fig. 2 the Auger detector was at $60^{\circ}$. The satellite-state coincidence signal that forms the continuum background is about a factor of 20 higher in the $60^{\circ}$ data than in the $90^{\circ}$ data. In each plot the line shape used in the fit is shown separately and normalized to emphasize the change in shape rather than the relative amplitude. The experimental data are the solid circles with error bars, and the fit results are shown as a solid line.

Figures 1(a) and 2(a) show data recorded for an excess energy of $22 \mathrm{eV}$ and a satellite-state separation energy of about $60 \mathrm{eV}$. The agreement between the theoretical prediction of PCI effects and the data of Fig. 1(a) is excellent. In Fig. 2(a) a slight asymmetry in the Fano fitting function gave the best result. Use of a Fano line shape in Fig. 2(a) is justified because even at such high separation energies the satellite-state coincidence background is

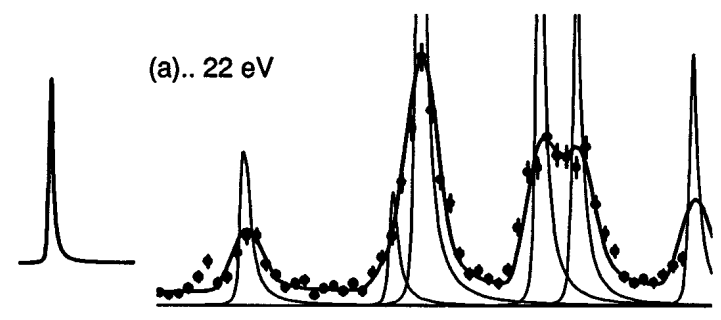

(b).. $2 \mathrm{eV}$
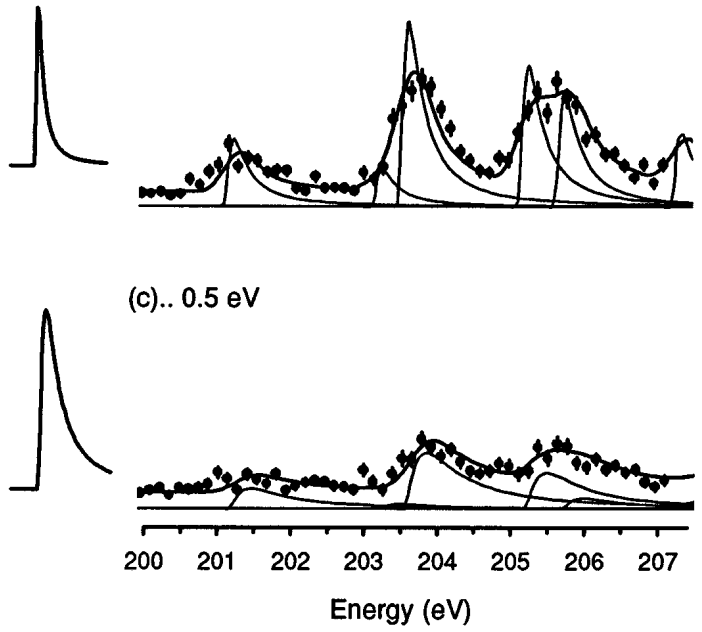

FIG. 1. Auger coincidence data obtained at $90^{\circ}$ for excess energies of (a) $22 \mathrm{eV}$, (b) $2 \mathrm{eV}$, and (c) $0.5 \mathrm{eV}$ above the $L_{3}$ ionization threshold. The PCI line shape used to fit the data is shown separately. The Auger lines are shown at the corresponding energies. Their sum is convoluted with a suitable Gaussian spectrometer function and shown as the solid line. The maximum intensity on the linear scale is 350 . 

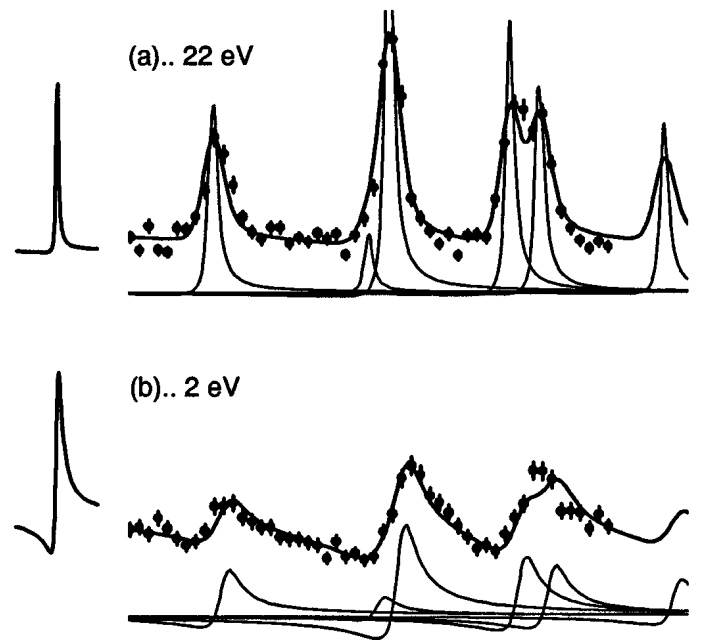

(c).. $0.5 \mathrm{eV}$

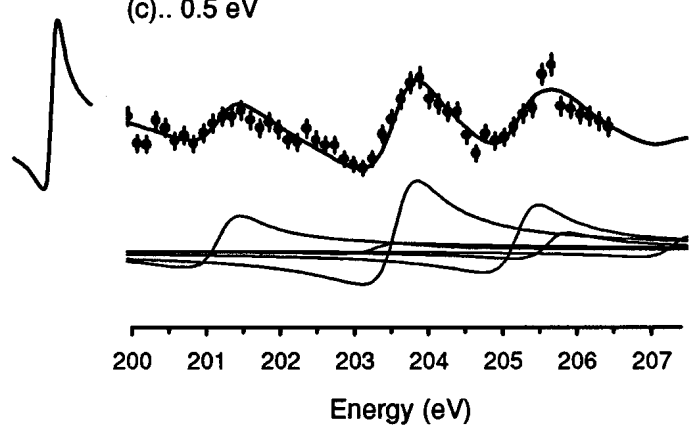

FIG. 2. Same as Fig. 1 except the Auger detection angle was $60^{\circ}$ and the fit is made with a Fano line shape that includes the predicted PCI effects.

nonzero. The Fano model included the shift and broadening predicted by the PCI model.

Closer to the $L_{2,3}$ ionization threshold, Figs. 1(b) and 2(b) show data for $2 \mathrm{eV}$ excess energy. In Fig. 1(b) the predicted PCI line shapes at $90^{\circ}$ are again in very good agreement with the experimental data, and there is no evidence to suggest that interference is significant. In comparison, the satellite background is appreciable at $60^{\circ}$, and this is reflected in the amount of interference observed in Fig. 2(b). With the observed PCI shift and broadening included, the fitted Fano line shape shows noticeable asymmetry. The fitted line shape suggests a slightly smaller degree of interference than indicated by the line shape fitted at this excess energy by Lohmann [8]. This may be because our method of data acquisition gives a better indication of the continuum background intensity. The good fit of the Fano line shape model shown in Fig. 2(b) at $60^{\circ}$ could not be reproduced by fitting only a PCI line shape to the same data.

The data for $0.5 \mathrm{eV}$ excess energy are shown in Figs. 1(c) and 2(c). Since the excess energy for $L_{2}$ transitions would be less than zero for this data, their intensity was adjusted to reflect the relative intensity expected due to the finite excess energy resolution. The agreement between our data and the PCI theory in Fig. 1(c) is still very good. A similar consideration was made when fitting the data of Fig. 2(c). The fitted Fano line shape that includes the predicted PCI effects indicates an even greater degree of interference than for $2 \mathrm{eV}$ excess energy.

For near-zero excess energy the separation energy of the argon satellite states is about $40 \mathrm{eV}$ and the large coincidence background is attributed mainly to the $3 s^{2} 3 p^{4}\left({ }^{1} D\right) n d{ }^{2} S(n=3,4,5)$ satellite states [12]. The satellite ionization process is more likely than direct double ionization, and leads to a final $\mathrm{Ar}^{+}$ion state. During the satellite ionization process one electron was ejected into the continuum, and another was promoted to an outer shell (mostly to " $n d$ " orbitals). The final ion is left with two vacancies in the $M_{2,3}$ orbitals. If an $L_{2,3} M_{2,3} M_{2,3}$ Auger electron is produced when the excess energy is small and the ejected electron escapes into the continuum but is recaptured by the ion, this also leaves an $\mathrm{Ar}^{+}$ion with two vacancies in the $M_{2,3}$ orbitals, with the captured electron occupying a weakly bound state. Recapture near the $L_{2,3}$ ionization threshold would enhance the $\mathrm{Ar}^{+}$ion yield at the expense of the $\mathrm{Ar}^{2+}$ yield in a similar way to that observed [13] for photoionization near the innershell ionization threshold. The probability of recapture is increased when the "slow" electron loses energy in the PCI process. If the captured electron is bound into a state with $n<6$, it is less likely to autoionize [14], and the final configuration may be identical to that produced by satellite-state ionization. For $(e, 2 e)$ detection purposes a recapture event is therefore identical to a satellite-state ionization. The observed interference is therefore more likely to be due to the effects of electron recapture leading to the same final $\mathrm{Ar}^{+}$configurations rather than autoionization of the satellite states to produce $\mathrm{Ar}^{2+}$ [6].

The most interesting interference data were recorded at $60^{\circ}$ for energies that correspond to Auger electron production below the $L_{3}$ ionization threshold. An example of this is shown in Fig. 3 for data $3 \mathrm{eV}$ below the $L_{3}$ threshold. This is low enough that the data should contain negligible contributions from above-threshold excess energies due to the detector resolution of $\pm 1.3 \mathrm{eV}$. At $90^{\circ}$ the satellitestate cross section was still small, and the data could be fitted using only a uniform flat background. There was no evidence above the statistics of the coincidence data of the expected resonant Auger transitions below threshold [15] at $90^{\circ}$. In contrast, Fig. 3 shows there was significant structure below threshold in the data at $60^{\circ}$. Figure 3(a) is a Fano line shape fit that includes the predicted PCI shift and broadening for an excess energy of $0.3 \mathrm{eV}$. This excess energy was the highest that might make even a slight contribution to the observed data because of the finite energy resolution, and it could probably be reduced, although this would increase the energy shift and make the line even broader, and the fit would become worse. It is clear from Fig. 3(a) that inclusion of PCI effects below threshold makes a reasonable fit almost impossible, due mostly to the energy shift component. Figure 3(b) shows that a pure Fano line shape, with no PCI shift or broadening, gives 

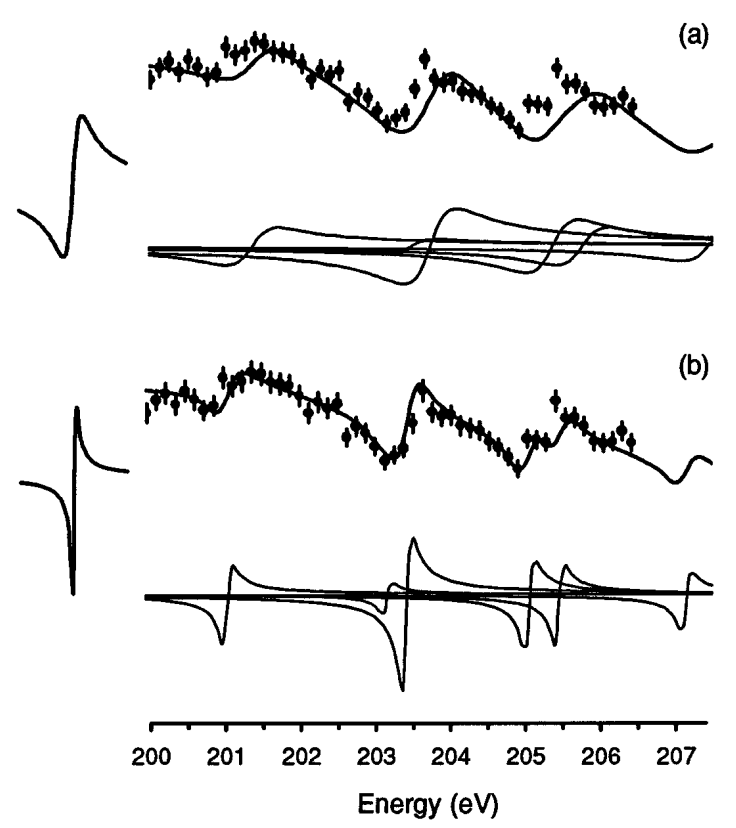

FIG. 3. Auger coincidence data obtained at $60^{\circ}$ and $3 \mathrm{eV}$ below the $L_{3}$ ionization threshold. The data are fitted in (a) using a Fano line shape that includes the PCI shift and broadening effects, and in (b) by a Fano line shape that does not include PCI effects.

an excellent fit to the same data. It also indicates the interference is greatest below the $L_{2,3}$ ionization threshold. The energies are the nominal $L_{2,3} M_{2,3} M_{2,3}$ Auger transition energies. This might suggest that resonant Auger transitions, the strongest of which occur within $1.7 \mathrm{eV}$ of the $L_{3}$ threshold [15], and with quite different energies, do not contribute much to the interference. Small interference effects, attributed to interference between the direct ionization process and resonant Auger decay leading to the same final state were recently reported [16] in constantionic-state photoionization Auger spectra recorded near the $2 p_{3 / 2}^{-1} 3 d$ threshold. Values of the fitted parameters for a wide range of excess energies will be given in a future publication.

To be consistent with our observations in the data of Fig. 3 of no PCI effects below threshold, the "ejected" electron would have to remain within the ion, at least until the Auger electron was emitted. This would explain why a Fano line shape could be fitted below threshold without including PCI effects, and like the recapture process, the final ion would be $\mathrm{Ar}^{+}$. However, as described above, the energies of the observed features suggest they are not resonant Auger transitions in the conventional sense. In this coincidence experiment the fast scattered electron and the Auger electron are not distinguishable from the products of a direct satellite-state ionization, and most probably this is the cause of the observed interference.

Finally, following electron-impact ionization, the $(e, 2 e)$ coincidence PCI line shift of several of the argon $L_{2,3} M_{2,3} M_{2,3}$ Auger transitions was observed at a number of excess energies. The observations are in excellent agreement with current theoretical calculations [3] for conditions where the coincidence background due to outer-shell satellite states is small. Interference between the Auger and satellite-state processes was observed when the satellite background was appreciable. Analysis has clearly shown that the amount of interference increases as the excess energy decreases towards threshold, and even below threshold. The interference originates with processes that can lead to the same final states as those produced by satellite-state ionization processes. For $L_{2,3}$ ionization this may occur when the slow electron is excited within the ion rather than ejected into the continuum, or when the slow electron that was ejected into the continuum with near-threshold energy is recaptured. This latter possibility may occur particularly when the ejected electron loses energy via PCI with the Auger electron.

This work was supported by the Australian Research Council and the University of Western Australia. The authors would like to thank Dr. Peter Hayes, Dr. Andrew Mikosza, and Mr. John Flexman for their contributions to this work.

[1] U. Fano, Phys. Rev. 124, 1866 (1961).

[2] B. Lohmann, Aust. J. Phys. 49, 365 (1996), and references therein.

[3] S. A. Sheinerman, W. Kuhn, and W. Mehlhorn, J. Phys. B 27, 5681 (1994).

[4] H. Ishii et al., Phys. Rev. A 43, 134 (1991), and references therein.

[5] E. C. Sewell and A. Crowe, J. Phys. B 17, 2913 (1984).

[6] W. Sandner and M. Volkel, J. Phys. B 17, L597 (1984).

[7] G. Stefani, L. Avaldi, A. Lahmam-Bennani, and A. Duguet, J. Phys. B 19, 3787 (1986).

[8] B. Lohmann, J. Phys. B 24, L249 (1991).

[9] B. W. Shore, J. Opt. Soc. Am. 57, 881 (1967).

[10] D. K. Waterhouse, J.F. Williams, and P. A. Smith, Rev. Sci. Instrum. 67, 1769 (1996), and references therein.

[11] W. Sandner and M. Völkel, Phys. Rev. Lett. 62, 885 (1989).

[12] I. E. McCarthy, R. Pascual, P. Storer, and E. Weigold, Phys. Rev. A 40, 3041 (1989).

[13] J. A. R. Samson et al., Phys. Rev. A 54, 2099 (1996).

[14] G. B. Armen and F. P. Larkins, J. Phys. B 25, 931 (1992).

[15] J. A. de Gouw et al., J. Phys. B 28, 2127 (1995).

[16] R. Camilloni et al., Phys. Rev. Lett. 77, 2646 (1996). 